\title{
One-pot synthesis of quinaldine derivatives by using microwave irradiation without any solvent - A green chemistry approach
}

\author{
JAVAD SAFARI*, SAYED HOSSEIN BANITABA and SEPEHR SADEGH SAMIEI \\ Department of Chemistry, The Faculty of sciences, University of Kashan, Kashan, \\ P.O. Box 87317-51167, I.R. Iran \\ e-mail: safari@kashanu.ac.ir; banitaba@grad.kashanu.ac.ir
}

MS received 19 October 2008; revised 23 February 2009; accepted 3 March 2009

\begin{abstract}
A convenient and efficient procedure for synthesis of quinaldine derivatives has been developed by a simple one-pot reaction of aniline derivatives and acetaldehyde on the surface of neutral alumina impregnated with hydrochloric acid under microwave irradiation without any solvent according to green chemistry.
\end{abstract}

Keywords. Quinaldine; one-pot; microwave irradiation; solvent-free; green chemistry; brønsted acid.

\section{Introduction}

Quinoline and their derivatives are important constituents of biologically active synthetic compounds, ${ }^{1-6}$ as several derivatives of this hydrocyclic unit have been found to posses useful biological activities such as antimicrobial, ${ }^{7}$ antimalarial, ${ }^{8}$ antibacterial. ${ }^{9,10}$ Many synthetic methods such as Skraup, ${ }^{11}$ DoebnerVon Miller, ${ }^{12}$ Friedlander, ${ }^{13}$ Combes reactions have been developed for the preparation of quinolines. A number of other methods have been reported for the synthesis of quinolines involving a variety of metal catalysts and Lewis acids. However, many of these procedures are not fully satisfactory with regard to operational simplicity, cost of the reagent and isolated yield. Thus, the drive continues to find a better and improved methodology. One-pot syntheses that involve environmental friendly chemical processes, and economically viable have received attention as a next strategy for 'green' organic syntheses. ${ }^{14}$ Recently, much attention has been focused on microwaveassisted organic reactions in the absence of solvent. ${ }^{15}$ Often, thermal demanding reactions take hours in solution, and may require repetitive treatments with excess reagents to drive them to completion. However, with microwave irradiation these same reactions may be completed in minutes. 15,16

Here we have demonstrated procedure for synthesis of quinaldines by one-pot reaction of anilines and

*For correspondence acetaldehyde, under microwave irradiation without any solvent (figure 1).

\section{Experimental}

\subsection{General}

Chemicals were purchased from Fluka, Merck and Aldrich chemical companies. Melting points were determined in open capillaries using an Electrothermal Mk3 apparatus. IR spectra were recorded using a Perkin-Elmer FT-IR 550 spectrophotometer. ${ }^{1} \mathrm{H}$ NMR and ${ }^{13} \mathrm{C}$ NMR spectra were recorded on a Bruker DRX-500 spectrometer for sample as indicated with tetramethylsilane as internal reference. UV spectra were recorded on a Hitachi 200-20 spectrophotometer using spectrophotometeric grade chlorform (Baker). MS spectra were recorded on a Finnigan MAT 44S, with an ionization voltage of $70 \mathrm{eV}$. The element analyses $(\mathrm{C}, \mathrm{H}, \mathrm{N})$ were obtained from a Carlo ERBA Model EA 1108 analayser carried out on Perkin- Elmer 240c analyzer, their results were found to be in good agreement $( \pm 0 \cdot 2 \%)$

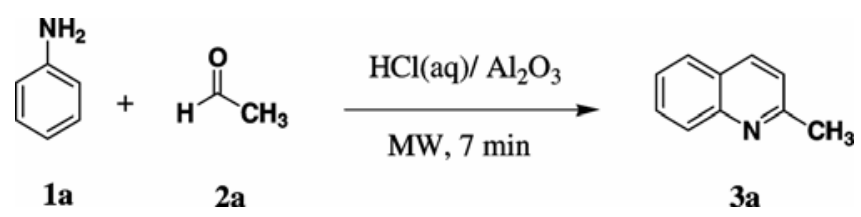

Figure 1. Synthesis of quinaldine. 
with the calculated values. Yields refer to isolated products.

\subsection{General synthesis of quinaldine derivatives}

The preparation of quinaldine (3a), is illustrative of the general procedure employed. To a mixture of 1 $(1 \mathrm{mmol}, 143 \mathrm{mg})$ and $2(2.4 \mathrm{mmol}, 105.7 \mathrm{mg})$ placed in an open glass container, alumina impregnated with concentrate hydrochloric acid (aq.) was added and the reaction mixture was irradiated in a microwave oven (ETHOS 1600, Milestone) with a power of $600 \mathrm{~W}$ for $7 \mathrm{~min}$. Upon completion of the reaction, as followed by TLC, the product purified by column chromatography (10\% ethyl acetate $/ 90 \%$ petroleum ether). Evaporation of the solvent gave pure quinaldine in $88 \%$ yield (cf. table 2 ).

3.2a 6-Methoxy-2,8-dimethylquinoline (3j, $\left.\mathrm{C}_{12} \mathrm{H}_{13} \mathrm{NO}\right)$ : Yield: $96 \%$, m.p.: $72-75^{\circ} \mathrm{C}$. IR $\left(\mathrm{KBr}, \mathrm{cm}^{-1}\right): 3000$ $(\mathrm{C}-\mathrm{H}), 1608(\mathrm{C}=\mathrm{N}), 1570(\mathrm{C}=\mathrm{N}), 1115(\mathrm{C}-\mathrm{O}) \mathrm{cm}^{-1}$. ${ }^{1} \mathrm{H}$ NMR $\left(500 \mathrm{MHz}, \mathrm{CDCl}_{3}\right): \delta 2 \cdot 9(s, 3 \mathrm{H}, \mathrm{Me}), 3 \cdot 1$ $(s, 3 \mathrm{H}, \mathrm{Me}), 3.9(s, 3 \mathrm{H}, \mathrm{OMe}), 6.9(d, 1 \mathrm{H}$, $\left.{ }^{4} J=2 \cdot 1 \mathrm{~Hz}\right), 7.5\left(d, 1 \mathrm{H},{ }^{4} J=2.1 \mathrm{~Hz}\right), 7.4(d, 1 \mathrm{H}$, $\left.{ }^{3} J=8 \cdot 1 \mathrm{~Hz}\right), 7 \cdot 3\left(d, 1 \mathrm{H},{ }^{3} J=8 \cdot 1 \mathrm{~Hz}\right) \mathrm{ppm} \cdot{ }^{13} \mathrm{C}$ NMR $\left(125 \mathrm{MHz}, \mathrm{CDCl}_{3}\right): \delta 19.4\left(\mathrm{CH}_{3}\right), 25.3\left(\mathrm{CH}_{3}\right), 57.7$ (OMe), 111.3 (CH), 123.1 (CH), 124.5 (CH), 126.6 (CH), 127.9 (C), 138.6 (C), 144.1 (C), 156.7 (C), 158.4 (C) $\mathrm{ppm}$. MS $(70 \mathrm{eV}) \mathrm{m} / z=187\left(\mathrm{M}^{+}, 100\right)$, $188,155,130$. UV $(\mathrm{EtOH}): \lambda_{\max }(\varepsilon)=267,302$, $315 \mathrm{~nm}$. Anal. Calcd. for $\mathrm{C}_{12} \mathrm{H}_{13} \mathrm{NO}: \mathrm{C}, 76.98 ; \mathrm{H}$, 7.00; N, 7.48. Found: C, 76.89; H, 6.93; N, 7.41.

3.2b 8-Methoxy-2,6-dimethylquinoline (3k, $\left.\mathrm{C}_{12} \mathrm{H}_{13} \mathrm{NO}\right)$ : Yield: $93 \%$, m.p.: $120-122^{\circ} \mathrm{C}$. IR $\left(\mathrm{KBr}, \mathrm{cm}^{-1}\right): 2995$ $(\mathrm{C}-\mathrm{H}), 1615(\mathrm{C}=\mathrm{N}), 1535(\mathrm{C}=\mathrm{C}), 1108(\mathrm{C}-\mathrm{O}) \mathrm{cm}^{-1}$. ${ }^{1} \mathrm{H}$ NMR $\left(500 \mathrm{MHz}, \mathrm{CDCl}_{3}\right): \delta 3 \cdot 2(s, 3 \mathrm{H}, \mathrm{Me}), 2 \cdot 4$ $(s, 3 \mathrm{H}, \mathrm{Me}), 4 \cdot 2(s, 3 \mathrm{H}, \mathrm{OMe}), 6.8\left(d, 1 \mathrm{H},{ }^{4} J=\right.$ $2.2 \mathrm{~Hz}), 7.1 \quad\left(d, 1 \mathrm{H},{ }^{4} J=2.2 \mathrm{~Hz}\right), 7.4 \quad(d, 1 \mathrm{H}$, $\left.{ }^{3} J=8.6 \mathrm{~Hz}\right), 8 \cdot 1\left(d, 1 \mathrm{H},{ }^{3} J=8.6 \mathrm{~Hz}\right) \mathrm{ppm} .{ }^{13} \mathrm{C}$ NMR $\left(125 \mathrm{MHz}, \mathrm{CDCl}_{3}\right): \delta 22.7\left(\mathrm{CH}_{3}\right), 25.9\left(\mathrm{CH}_{3}\right), 56.6$ $(\mathrm{OMe}), 113.2(\mathrm{CH}), 123.5(\mathrm{CH}), 124.8(\mathrm{CH}), 125.9$ (CH), 128.7 (C), 143.6 (C), 144.7 (C), 151.0 (C), 156.2 (C) $\mathrm{ppm}$. MS $(70 \mathrm{eV}) \mathrm{m} / z=187\left(\mathrm{M}^{+}, 100\right)$, $188,155,130$. UV $(\mathrm{EtOH}): \lambda_{\max }(\varepsilon)=268,301$, $221 \mathrm{~nm}$. Anal. Calcd. for $\mathrm{C}_{12} \mathrm{H}_{13} \mathrm{NO}$ : C, 76.98; $\mathrm{H}$, 7.00; N, 7.48. Found: C, 76.89; H, 6.93; N, 7.41.

3.2c 8-Methoxy-2-methyl-5-nitro-6-quinolinamine (31, $\mathrm{C}_{11} \mathrm{H}_{11} \mathrm{~N}_{3} \mathrm{O}_{3}$ ): Yield: $89 \%$, m.p.: $179-183^{\circ} \mathrm{C}$.
IR $\left(\mathrm{KBr}, \mathrm{cm}^{-1}\right): 2999(\mathrm{C}-\mathrm{H}), 1610(\mathrm{C}=\mathrm{N}), 1640$, $1569(\mathrm{C}=\mathrm{C}), 1540(\mathrm{~N}=\mathrm{O}), 1353(\mathrm{~N}=\mathrm{O}), 1111(\mathrm{C}-\mathrm{O})$ $\mathrm{cm}^{-1} .{ }^{1} \mathrm{H}$ NMR $\left(500 \mathrm{MHz}, \mathrm{CDCl}_{3}\right): \delta 3 \cdot 1(s, 3 \mathrm{H}$, $\mathrm{Me}), 3.6(b, 2 \mathrm{H}, \mathrm{NH}), 4 \cdot 2(s, 3 \mathrm{H}, \mathrm{OMe}), 7 \cdot 2(d$, $\left.1 \mathrm{H}, \quad{ }^{3} J=8.4 \mathrm{~Hz}\right), \quad 7.5 \quad(s, \quad 1 \mathrm{H}), \quad 7.8 \quad(d, \quad 1 \mathrm{H}$, $\left.{ }^{3} J=8.4 \mathrm{~Hz}\right) \mathrm{ppm} .{ }^{13} \mathrm{C}$ NMR $\left(125 \mathrm{MHz}, \mathrm{CDCl}_{3}\right): \delta$ $23.9\left(\mathrm{CH}_{3}\right), 57.0(\mathrm{OMe}), 110.5(\mathrm{CH}), 116.3(\mathrm{C})$, $124.8(\mathrm{CH}), 132.3(\mathrm{CH}), 134.7$ (C), $135.9(\mathrm{C}), 144.5$ (C), 155.2 (C), 160.2 (C) ppm. MS (70 eV) $\mathrm{m} / \mathrm{z}=$ $233\left(\mathrm{M}^{+}, 100\right), 202,186,143$. UV $($ EtOH $): \lambda_{\max }(\varepsilon)=$ 253, 303, $233 \mathrm{~nm}$. Anal. Calcd for $\mathrm{C}_{11} \mathrm{H}_{11} \mathrm{~N}_{3} \mathrm{O}_{3}$ : C, $56.65 ; \mathrm{H}, 4.75$; N, 18.02. Found: C, 56.56; H, 4.67; $\mathrm{N}, 17.95$.

\section{$3.2 \mathrm{~d}$ 6,8-Dichloro-5-methoxy-2-methylquinoline}

$\left(3 \mathrm{~m}, \mathrm{C}_{11} \mathrm{H}_{9} \mathrm{Cl}_{2} \mathrm{NO}\right)$ : Yield: $80 \%$, m.p.: $231-234^{\circ} \mathrm{C}$. IR $\left(\mathrm{KBr}, \mathrm{cm}^{-1}\right): 3000(\mathrm{C}-\mathrm{H}), 1587(\mathrm{C}=\mathrm{N}), 1520$ $(\mathrm{C}=\mathrm{C}), 1119(\mathrm{C}-\mathrm{O}), 1103(\mathrm{C}-\mathrm{Cl}) \mathrm{cm}^{-1} .{ }^{1} \mathrm{H}$ NMR $\left(500 \mathrm{MHz}, \mathrm{CDCl}_{3}\right): \delta 3 \cdot 3(s, 3 \mathrm{H}, \mathrm{Me}), 3 \cdot 8(s, 3 \mathrm{H}$, OMe $), 6.9\left(d, 1 \mathrm{H},{ }^{3} J=9.0 \mathrm{~Hz}\right), 7.7(s, 1 \mathrm{H}), 8 \cdot 1(d$, $\left.1 \mathrm{H},{ }^{3} \mathrm{~J}=9.0 \mathrm{~Hz}\right) \mathrm{ppm} .{ }^{13} \mathrm{C}$ NMR $\left(125 \mathrm{MHz}, \mathrm{CDCl}_{3}\right)$ : $\delta 24.4\left(\mathrm{CH}_{3}\right), 58.9(\mathrm{OMe}), 118.4(\mathrm{CH}), 119.8(\mathrm{CH})$, $122 \cdot 5$ (C), $124 \cdot 4$ (C), $127.5(\mathrm{C}), 128 \cdot 1(\mathrm{CH}), 146 \cdot 3$

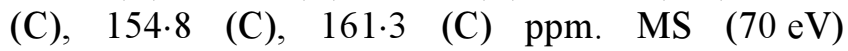
$m / z=242\left(\mathbf{M}^{+}, 100\right), 211,176,143$. UV (EtOH): $\lambda_{\max }(\varepsilon)=286, \quad 327, \quad 250 \mathrm{~nm}$. Anal. Calcd. for

Table 1. The yields for the synthesis of quinaldine 3a with different Brønsted acids under microwave irradiation.

\begin{tabular}{llcc}
\hline Entry & Brønsted acid & Equivalent & Yield (\%) \\
\hline 1 & $\mathrm{HCl}$ & $0 \cdot 7$ & 32 \\
2 & $\mathrm{HCl}$ & 1 & 54 \\
3 & $\mathrm{HCl}$ & $1 \cdot 5$ & 60 \\
4 & $\mathrm{HCl}$ & $1 \cdot 8$ & 80 \\
5 & $\mathrm{HCl}$ & $2 \cdot 2$ & 90 \\
6 & $\mathrm{H}_{2} \mathrm{SO}_{4}$ & $2 \cdot 2$ & 83 \\
7 & $\mathrm{H}_{3} \mathrm{PO}_{4}$ & $2 \cdot 2$ & 28 \\
8 & $\mathrm{CH}_{3} \mathrm{CO}_{2} \mathrm{H}$ & $2 \cdot 2$ & Trace \\
\hline
\end{tabular}

Reaction conditions: 1a $(1 \mathrm{mmol}), 2$ a $(2.4 \mathrm{mmol})$, reaction time: $7 \mathrm{~min}$

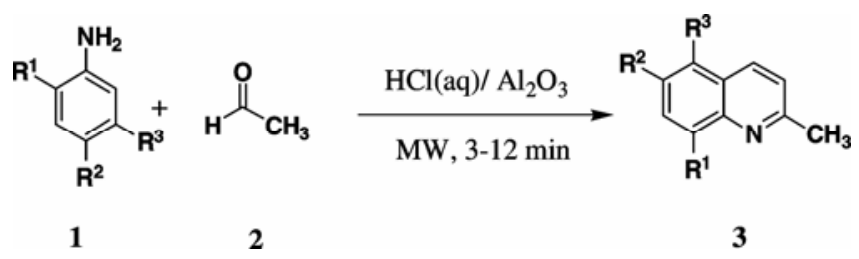

Figure 2. Synthesis of Quinaldine derivatives under microwave irradiation. 
Table 2. The yields for the synthesis of quinaldine derivatives produced by microwave irradiation.

\begin{tabular}{|c|c|c|c|c|c|c|c|c|}
\hline \multirow[b]{2}{*}{ Entry } & \multirow[b]{2}{*}{$\mathrm{R}^{1}$} & \multirow[b]{2}{*}{$\mathrm{R}^{2}$} & \multirow[b]{2}{*}{$\mathrm{R}^{3}$} & \multirow[b]{2}{*}{ Product } & \multirow[b]{2}{*}{ MW time (min) } & \multirow[b]{2}{*}{ Yield (\%) } & \multicolumn{2}{|c|}{ Mp. $\left({ }^{\circ} \mathrm{C}\right)$ or bp $\left({ }^{\circ} \mathrm{C}\right) /$ Torr } \\
\hline & & & & & & & Found & Reported \\
\hline 1 & $\mathrm{H}$ & $\mathrm{H}$ & $\mathrm{H}$ & $3 \mathbf{a}$ & 7 & 88 & $247-249^{\mathrm{a}}$ & $248^{12}$ \\
\hline 2 & $\mathrm{CH}_{3}$ & $\mathrm{H}$ & $\mathrm{H}$ & $\mathbf{3 b}$ & 9 & 79 & $26-29$ & $27^{17 a}$ \\
\hline 3 & $\mathrm{Cl}$ & $\mathrm{H}$ & $\mathrm{H}$ & $3 \mathbf{c}$ & 10 & 75 & $67-69$ & $68^{17 b}$ \\
\hline 5 & $\mathrm{NO}_{2}$ & $\mathrm{H}$ & $\mathrm{H}$ & 3d & 10 & Trace & - & \\
\hline 6 & $\mathrm{H}$ & $\mathrm{CH}_{3}$ & $\mathrm{H}$ & $3 \mathbf{e}$ & 5 & 91 & $59-61$ & $60^{17 a}$ \\
\hline 7 & $\mathrm{H}$ & $\mathrm{Cl}$ & $\mathrm{H}$ & $3 f$ & 10 & 89 & $91-94$ & $93^{17 b}$ \\
\hline 8 & $\mathrm{H}$ & $\mathrm{NO}_{2}$ & $\mathrm{H}$ & $3 g$ & 10 & 73 & $163-165$ & $165^{17 \mathrm{c}}$ \\
\hline 9 & $\mathrm{H}$ & $\mathrm{OCH}_{3}$ & $\mathrm{H}$ & $3 h$ & 5 & 95 & $62-64$ & $62-65^{17 d}$ \\
\hline 10 & $\mathrm{H}$ & $\mathrm{Br}$ & $\mathrm{H}$ & $3 \mathbf{i}$ & 10 & 90 & $101-103$ & $100^{17 \mathrm{e}}$ \\
\hline 11 & $\mathrm{CH}_{3}$ & $\mathrm{OCH}_{3}$ & $\mathrm{H}$ & $\mathbf{3 j}$ & 4 & 96 & $72-75$ & - \\
\hline 12 & $\mathrm{OCH}_{3}$ & $\mathrm{Me}$ & $\mathrm{H}$ & $3 \mathbf{k}$ & 5 & 93 & $120-122$ & - \\
\hline 13 & $\mathrm{OCH}_{3}$ & $\mathrm{NH}_{2}$ & $\mathrm{NO}_{2}$ & 31 & 3 & 89 & $179-183$ & - \\
\hline 14 & $\mathrm{Cl}$ & $\mathrm{Cl}$ & $\mathrm{OCH}_{3}$ & $3 m$ & 12 & 80 & $231-234^{\mathrm{a}}$ & - \\
\hline
\end{tabular}

${ }^{a}$ Numbers reported are boiling point
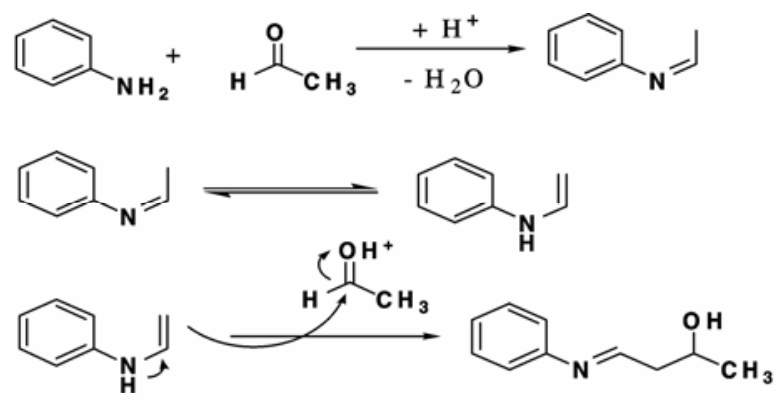<smiles>C/C=C/C=Nc1ccccc1</smiles><smiles>CC(C)=CC=Nc1ccccc1</smiles><smiles>CC1CC(Nc2ccccc2)C2(C=CC=CC2=NCCC[C@@H](C)Nc2ccccc2)Cc2ccccc21</smiles><smiles>CC1C=Cc2ccccc2N1</smiles><smiles>Cc1ccc2ccccc2n1</smiles>

Figure 3. The mechanism of quinaldine preparation.
$\mathrm{C}_{11} \mathrm{H}_{9} \mathrm{Cl}_{2} \mathrm{NO}: \mathrm{C}, 45 \cdot 57 ; \mathrm{H}, 3.75 ; \mathrm{N}, 5.79$. Found: $\mathrm{C}$, $45 \cdot 58 ; \mathrm{H}, 3 \cdot 66$; N, 5.69.

\section{Results and discussion}

We first choose 1a and searched for the best brønsted acid for its reaction with acetaldehyde $\mathbf{2 a}$ to afford quinaldine 3a. The yields and amounts of the used brønsted acid for this reaction of $\mathbf{1 a}$ with $\mathbf{2 a}$ were listed in table 1. Among the investigated acids, hydrochloric acid appeared to be the best catalyst for this reaction, showing the highest yield (table 1). Although sulfuric acid (table 1, entry 6) exhibited similar catalytic activity when compared with hydrochloric acid the latter was preferred because of its safety, more environmental friendliness and lower price. The above results prompted us to select 2.2 equiv. of hydrochloric acid as the catalyst for further study.

Typically, a mixture of $1(1.0 \mathrm{mmol})$ and 2 $(2.4 \mathrm{mmol})$ was added to alumina impregnated with hydrochloric acid. The whole mixture was stirred for 5 min for uniform mixing and was then irradiated by microwave oven (ETHOS 1600, Milestone) with a power of $600 \mathrm{~W}$ specially designed for organic synthesis (figure 2). After the reaction, the product was purified by column chromatography $(10 \%$ ethyl acetate $/ 90 \%$ petroleum ether). The results are presented in table 2 .

A wide range of substituted anilines were subjected to this procedure to produce the corresponding qui- 
naldines. In general, the yields of quinaldines are not affected by the nature of substituents on the anilines (except entry 7). When 2-nitroaniline was used as a starting material, lead to decrease of yield in these procedures. According to the literature $^{18}$ the mechanism was proposed as shown in figure 3 .

Elimination of hazardous solvent and Lewis acid in this procedure and use from molecular oxygen as a green oxidant in place of other same reagent such as $\mathrm{I}_{2}$ and nitrobenzene to cause that, this procedure converted to safe and clean method, according to green chemistry. Advantages of this method include the fact that it is environmentally benign, economical and has a short reaction time.

\section{Conclusion}

In conclusion, we have demonstrated the procedure for preparation of quinaldines, from aniline derivatives and acetaldehyde under microwave irradiation without any solvent. The current method presents a simple and useful synthetic process for quinaldines because of the following advantages: (i) use of cheap and readily available hydrochloric acid, (ii) high yield and short reaction time, (iii) straightforward and easy work-up procedure, (iv) use of microwave irradiation as the novel source and efficient, (v) use of molecular oxygen as a green oxidant.

\section{Acknowledgements}

We are grateful to University of Kashan for financial support. We also thank Professor Brindabon C Ranu for scientific support.

\section{References}

1. Elguero J 1984 Comprehensive heterocyclic chemistry (ed.) A McKillop (Oxford: Pergamon Press) Vol. 5, p. 167

2. Penning T D, Talley J J, Bertenshaw S R, Carter J S, Colins P W, Graneton J M, Lee L F and Malecha J W 1997 J. Med. Chem. 401347

3. Menozzi G, Mosti L, Fossa P, Maltioli F and Ghia M 1997 J. Heterocycl. Chem. 34963

4. Naik R D and Desai K R 1998 Orient. J. Chem. 14161

5. Kidwai M and Negi N 1997 Monatsh. Chem. 12885

6. El-Sayed O A, El-Bieh F B, El-Aqeel S I, Al-Bassam B A and Hussein M E 2002 Bull. Chim. Farm. 141461

7. Selvi S T, Nadaraj V, Mohan S, Sasi R and Hema M 2006 Bioorg. Ed. Chem. 143896

8. Jashi A A, Narkhede S S and Viswanathan C L 2005 Bioorg. Med. Chem. Lett. 1573

9. Suresh T, Nandha Kumar R, Magesh S and Mohan P S 2003 Indian J. Chem. B42 2133

10. Suresh T, Nandha Kumar R, Magesh S and Mohan P S 2003 Indian J. Chem. B42 688

11. Skraup H 1880 Chem. Ber. 132086

12. Doebner O and Miller W 1883 Bericht. 162464

13. Friedlander P 1882 Chem. Ber. 152572

14. Hall N 1994 Science 26632

15. (a) Loupy A, Petit A, Hamelin J, Texier-Boullet F, Jacquault P and Mathe D 1998 Synthesis 2113; (b) Perreux L and Loupy A 2001 Tetrahedron 57 9199; (c) Lindstroem P, Tierney J, Wathey B and Westman J 2001 Tetrahedron 57 9225; (d) Varma R S 1999 Green Chem. 43

16. (a) Kim J K, Kwon P S, Kwon T W, Chung S K and Lee J W 1996 Synth. Commun. 26 535; (b) Kim S Y, Kwon P S, Kwon T W, Chung S K and Chang Y T 1997 Synth. Commun. 27 533, (c) Kwon P S, Kim Y S, Kang C J, Kwon T W, Chung S K and Chang Y T 1997 Synth. Commun. 274091

17. (a) Manske R H F 1942 Can. J. 20 133; (b) Minkin V I 1961 Chem. Abs. 55 14457; (c) Hamer F M $1921 \mathrm{~J}$. Chem. Soc. 119 1432; (d) Neumann M 1946 Org. Synth. 26 47; (e) Buu-Hoi N P 1948 J. Chem. Soc. 106110

18. Denmark S E and Venkatraman S $2006 \mathrm{~J}$. Org. Chem. 711668 